Scientific Review - Engineering and Environmental Sciences (2021), 30 (3), 496-508

Sci. Rev. Eng. Env. Sci. (2021), 30 (3)

Przegląd Naukowy - Inżynieria i Kształtowanie Środowiska (2021), 30 (3), 496-508

Prz. Nauk. Inż. Kszt. Środ. (2021), 30 (3)

http://iks.pn.sggw.pl

DOI 10.22630/PNIKS.2021.30.3.42

Marcel PAREDES ${ }^{1,2}$, Fausto LOPEZ ${ }^{1}$, Tito CASTILLO ${ }^{1}$, Alexis ANDRADE ${ }^{1,3}$, Valeria ARROBA ${ }^{1}$

${ }^{1}$ Universidad Nacional de Chimborazo, Facultad de Ingeniería

${ }^{2}$ Universidad Politécnica de Madrid, Escuela Técnica Superior de Ingenieros Industriales

${ }^{3}$ Universidad Politécnica de Valencia, Escuela Técnica Superior de Ingeniería de Caminos,

Canales y Puertos

\title{
Sustainable acoustic insulation for prefabricated concrete homes in Andean zones
}

Key words: Andean zones, acoustic insulators, construction, manufactured homes

\section{Introduction}

The use of precast concrete has become a viable way to build houses due to the high degree of reliability of its properties and characteristics when controlling the quality of the process and the quality standards in the production plant (du Plessis et al., 2021). This is corroborated by the precast division of the national cement union of Ecuador (Ucem), which based on basic criteria of high productivity at low cost, industrialization and applicability (low time for assembly), show that the properties that the concrete will develop are optimal in the face of variations due to on site work restric- tions (Kim, Kim \& Cho, 2020). During the design and selection of construction materials, the weather is a key factor. Sudden changes in temperature directly affect the behavior of the materials (creating cracks and/or detachments) due to thermal shock (Rajeshkumar, Anandaraj, Kavinkumar \& Elango, 2020). Due to its privileged geographical position, Ecuador has a variety of weather for its four regions: coast, Sierra, Amazon, and Galapagos; however, Andean zones presents the best conditions in terms of maintenance for the materials due to the cold temperatures (Cevallos, Jaramillo, Ávila \& Aldaz, 2017). Andean region is made of rows of mountains, snowcapped mountains, volcanoes, high Andean peaks, valleys, and lagoons. It is characterized by a humid tropical climate in transition zones towards the coast and 
the Amazon, temperate semi-humid to humid in the inter-Andean zone, hot and dry in the inter-Andean valleys and cold in the highlands and mountains in the paramos, above 3,000 $\mathrm{m}$ of altitude. Temperature is linked to altitude; between 1,500 and $3,000 \mathrm{~m}$ the average values vary between 8 and $20^{\circ} \mathrm{C}$, with a temperature gradient of $\sim 5^{\circ} \mathrm{C}$ for every 1,000 m of altitude (Narváez \& León, 2001). These climatic considerations are necessary when attempting to maintain comfort in habitability. This refers to construction solutions used in a building or home to minimize the transmission of sounds (noise or noise pollution) from the outside or from an adjacent room. This can be achieved using acoustic insulation materials (Escobar, 2019). The place of origin of the acoustic materials can be mineral or natural, synthetic, and bio-based. Through the years, both materials, techniques and technologies have improved, reaching a high acoustic quality of the constructions. Countries such as Spain or Ecuador are an excellent reference in terms of research on acoustic insulation of buildings (Taban et al., 2019). Even though the construction sector offers a variety of acoustic materials, it is unknown which are the most optimal materials to use them, due to the scarce information in Ecuador on the subject (Obaco, Royuela \& Matano, 2020). Consequently, it is necessary to prepare a comparative study of acoustic materials for prefabricated concrete homes for Andean zones of Ecuador. This research validates itself through its development: search and analysis of relevant updated academic and university research, data comparison and use of statistical tools and techniques that allow understanding and building a new research generating contexts; in this case on optimal acoustic materials for application in environments created with precast concrete (Guevara Patiño, 2016).

\section{Methodology}

The Delphi method was used for the determination of the best acoustic insulators, after the gathering of the information obtained by the experts, the results were validated with the AHP method (analytic hierarchy process), and organizing the information hierarchically and thus it was possible to determine the best materials for acoustic insulation in buildings in Andean area of Ecuador.

According to José, Vidal and Lluch (2019), the foundation of AHP is:

a) The structuring a hierarchical model (representation of the problem by identifying the goal, criteria, sub-criteria, and alternatives).

b) Prioritization of the elements of the hierarchical model.

c) Evaluation of the elements by assigning "weights".

d) Ranking of the alternatives according to the given weights.

e) Synthesis.

f) Analysis of sensitivity.

It is important to identify the problem in the methodology to accept the criteria of the experts and place the proposed alternatives on a list, allowing the use of these tools in solving the acoustic problem in Andean areas (Fig. 1). This will provide a series of viable solutions to builders and users. 


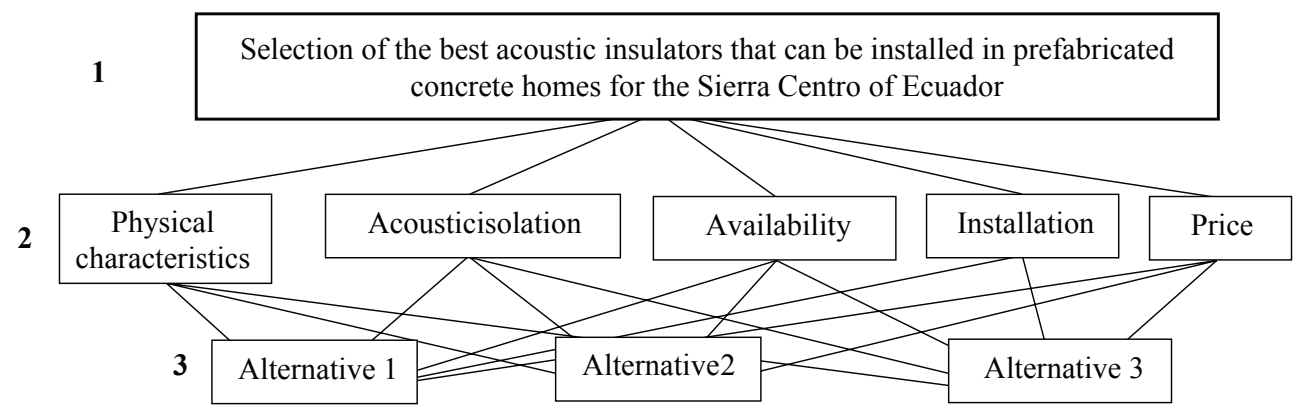

FIGURE 1. Hierarchy tree (García-Ruiz \& Lena-Acebo, 2018)

The main strategy of AHP is the paired comparisons, this uses an underlying scale with values from 1 to 9 to qualify the relative preferences of two elements, that is, a comparison is made between two elements to determine the order of the alternatives (Table 1).

The prioritization of the importance, preferences, or probabilities of pair elements. Based on a common attribute or criteria represented in the decision hierarchy, it is the mathematical basis of AHP (Chandna, Saini \& Kumar, 2021). In terms of quality to make the final decision, the consistency of the judgments shown during the series of paired comparisons is important. A perfect consistency is difficult to achieve, since in almost any set of paired comparisons there will always be some inconsistency; for they are judgments made by human beings. If the degree of consistency is acceptable this can be continued with the decision process, if the degree of consistency is unacceptable the decision maker will have to consider and possibly modify their judgement about the paired comparisons before continuing with the analysis. If the degree of consistency is acceptable this can be continued with the decision process, if the degree of consistency is unacceptable the decision maker will have to consider and possibly modify their judgement about the paired comparisons before continuing with the analysis.

To determine if the criteria are well evaluated, the consistency ratio $(C R)$

TABLE 1. Saaty scale for the AHP method (Lin \& Kou, 2021)

\begin{tabular}{|c|c|c|}
\hline Value & Definition & Comments \\
\hline 1 & same importance & Criteria A is equal to the importance of criteria B. \\
\hline 3 & moderated importance & Experience and judgment slightly favor criteria A over criteria B. \\
\hline 5 & big importance & Experience and judgment strongly favor criteria A over criteria B. \\
\hline 7 & very big importance & Criteria A is much more important than criteria B. \\
\hline 9 & extreme importance & The greater importance of criteria A over criteria B is beyond \\
doubt.
\end{tabular}


calculation is used, where the result of the criteria is compared with the data shown in Table 2.

TABLE 2. Maximum percentage of consistency ratio

\begin{tabular}{|c|c|}
\hline Matrix size & $\%$ max. $C R$ \\
\hline 3 & $5 \%$ \\
\hline 4 & $9 \%$ \\
\hline$\geq 5$ & $10 \%$ \\
\hline
\end{tabular}

Matrix size formulas used to calculate consistency ratio:

$I C=\left(\lambda \_-n\right) /(n-1)$

$I A=(1.98 \cdot(n-2)) / n$

$C R=I C / I A$

where:

$I C$ - consistency index,

$C R$ - consistency ratio,

$\lambda p$ - average Landa,

$I A$ - Ranm consistency,

$n$ - array order.

Note: Landa is the quotient of the row vector and the average vector of the material comparison matrix; average Landa $(\lambda, p)$ is needed to evaluate the consistency of the evaluations made by the experts (Caner \& Aydin, 2021).

\section{Results and discussion}

Based on a bibliographic search on the different types of acoustic insulators, it was observed that, for an acoustic insulating material, there are no parameters defined by any regulations. However, there are some characteristics that can help to compare and choose an optimal material depending on the type of noise to be mitigated (Dong et al., 2021). In general, it is understood as "acoustic insulation" to reduce the noise level of a room (Opydo, 2004). However, the difference between the terms "isolation" and "absorption" must be considered. The acoustic absorbers are used to being materials of low density (they are light) and great flexibility. Acoustic insulators, on the other hand, are materials of high density (heavy) and greater rigidity, for each type of noise there are certain physical characteristics that the materials must meet so that their use contributes to noise reduction (Sato, Kurisu, Morimoto \& Maeda, 2021). In the same manner, as the chosen insulation will be applied in the Sierra del Ecuador area in height ranges from 2,000 to $6,700 \mathrm{~m}$ above sea level, relevant characteristics must be considered in terms of functionality; more specifically to the data on the coefficient of thermal expansion (Bao et al., 2021). Table 3 shows the value of this characteristic of the materials and shows characteristics to be considered for the functionality of the material.

The characteristics of the acoustic insulating / absorbent materials, shown in Table 3, serve as a guide for an adequate comparison between acoustic insulating / / absorbent materials. It is worth mentioning that most of the bibliography, consulted publications, do not fully specify the physical characteristics of the tested materials (thickness, density, porosity, among others).

On the other hand, the value of the thermal shock coefficient represents the expansion of the volume of the material; here, materials shrink in cold and expand in heat (He, Huang \& Jiang, 2021). The lower the coefficient of 


\begin{tabular}{|c|c|c|c|c|c|c|c|c|c|c|c|}
\hline 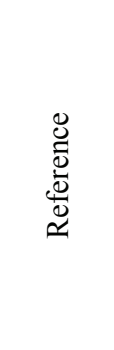 & 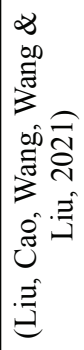 & 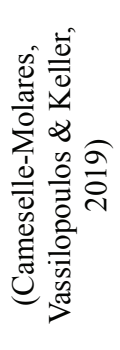 & 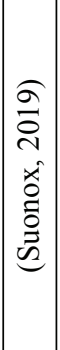 & 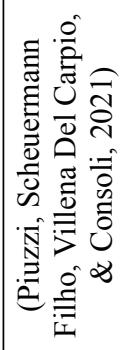 & 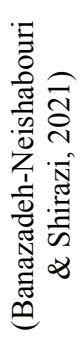 & 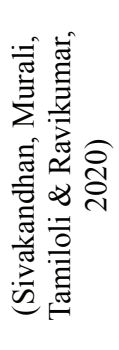 & 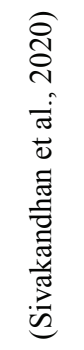 & 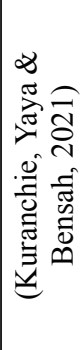 & 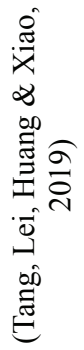 & 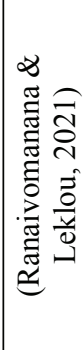 & 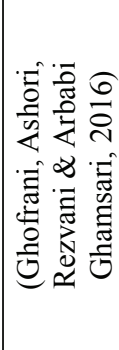 \\
\hline 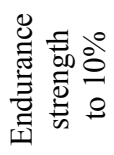 & $\frac{\sum^{\sigma}}{\dot{0}}$ & $\frac{\ll}{z}$ & 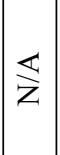 & 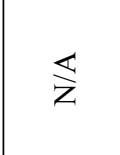 & 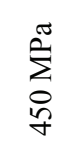 & $\underset{\mathrm{z}}{\varangle}$ & $\frac{\nwarrow}{Z}$ & $\sum_{0}^{\infty}$ & $\frac{\pi}{8}$ & $\frac{\pi}{\frac{\sigma}{\delta}}$ & $\frac{\ll}{Z}$ \\
\hline 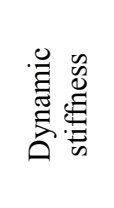 & 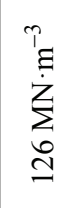 & $\underset{z}{\varangle}$ & 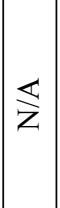 & 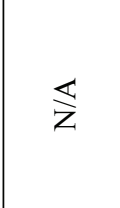 & $\underset{乙}{\varangle}$ & 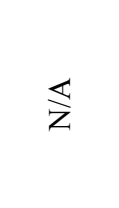 & $\frac{\nwarrow}{Z}$ & 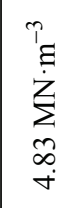 & 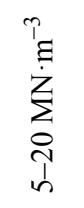 & $\frac{\nwarrow}{Z}$ & $\underset{Z}{\varangle}$ \\
\hline 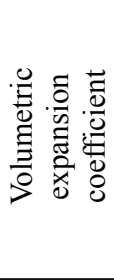 & 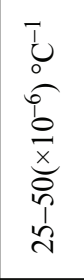 & $\underset{z}{\varangle}$ & 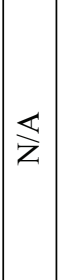 & 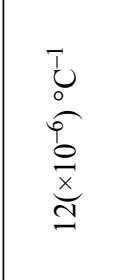 & 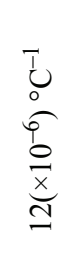 & $\underset{乙}{\varangle}$ & $\underset{Z}{\nwarrow}$ & $\begin{array}{l}\vec{b} \\
0 \\
\sigma \\
0 \\
\underline{x} \\
\infty \\
i \\
n\end{array}$ & $\begin{array}{l}1 \\
\vdots \\
0 \\
\sigma \\
0 \\
\frac{x}{x} \\
0 \\
\vec{\tau} \\
\vdots \\
0\end{array}$ & 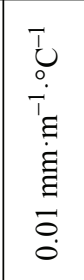 & $\underset{Z}{\nwarrow}$ \\
\hline 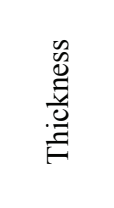 & $\begin{array}{l}\Xi \\
\Xi \\
\stackrel{\Xi}{\circ}\end{array}$ & 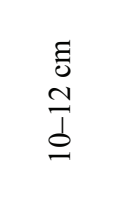 & 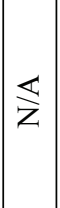 & $\begin{array}{l}\Xi \\
\Xi \\
n \\
\infty \\
\sim\end{array}$ & 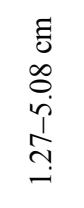 & 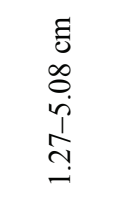 & 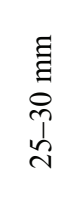 & $\begin{array}{l}\tilde{y} \\
\text { ñ }\end{array}$ & 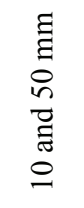 & $\begin{array}{l}\text { ह } \\
\text { స̃ } \\
\text { bे }\end{array}$ & $\begin{array}{c}\Xi \\
\Xi \\
\text { ป }\end{array}$ \\
\hline 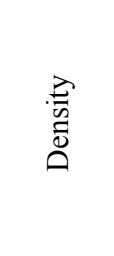 & 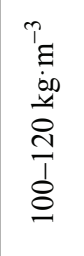 & 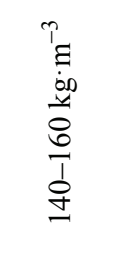 & 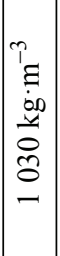 & 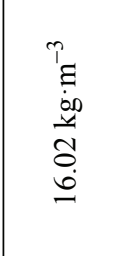 & 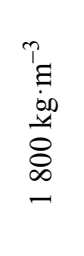 & $\frac{\nwarrow}{Z}$ & 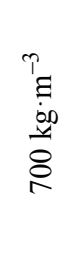 & 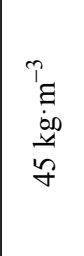 & 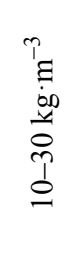 & 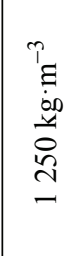 & $\underset{乙}{\varangle}$ \\
\hline 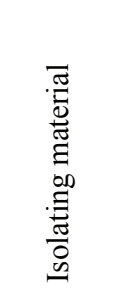 & 产 & 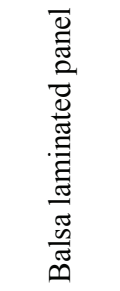 & 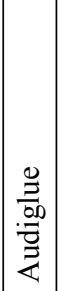 & 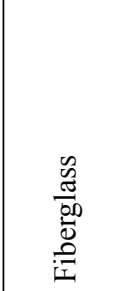 & 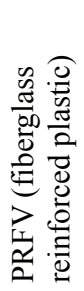 & 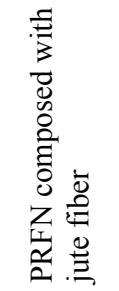 & 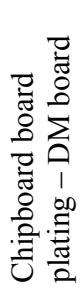 & 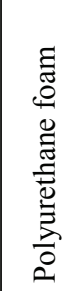 & 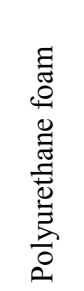 & 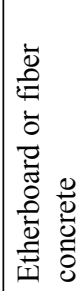 & 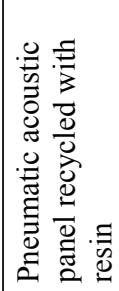 \\
\hline
\end{tabular}




\begin{tabular}{|c|c|c|c|c|c|c|c|c|c|c|c|c|}
\hline 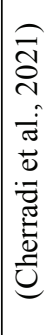 & 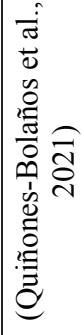 & 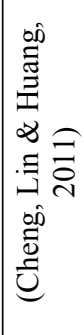 & 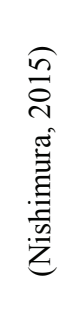 & 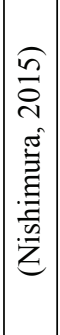 & 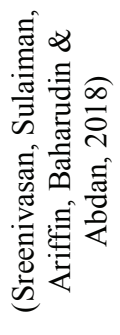 & 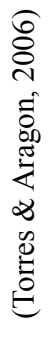 & 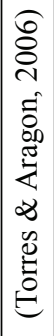 & 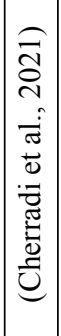 & 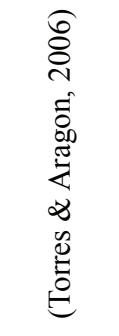 & 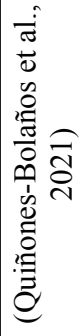 & 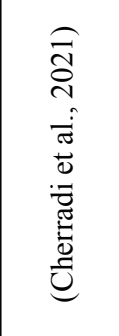 & 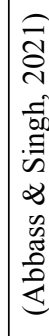 \\
\hline$\frac{\tilde{\sigma}}{\stackrel{0}{8}}$ & $\frac{\nwarrow}{Z}$ & $\frac{\sqrt[\pi]{7}}{\frac{\pi}{\sigma}}$ & $\underset{z}{\varangle}$ & $\frac{\frac{2}{y}}{8}$ & $\underset{z}{\varangle}$ & $\underset{z}{Z}$ & 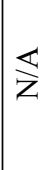 & $\mid \begin{array}{c}1 \\
\vdots \\
\dot{1} \\
8 \\
0 \\
1 \\
0 \\
n\end{array}$ & $\underset{z}{\varangle}$ & $\underset{z}{Z}$ & $\underset{z}{\overparen{Z}}$ & $\overleftrightarrow{\text { Z }}$ \\
\hline$\overleftrightarrow{\mathrm{z}}$ & $\underset{\mathrm{Z}}{\overleftrightarrow{\mathrm{Z}}}$ & $\underset{z}{\varangle}$ & 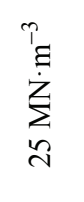 & 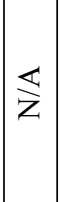 & $\underset{z}{\varangle}$ & $\underset{\mathrm{z}}{\overleftrightarrow{\mathrm{z}}}$ & 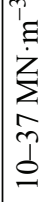 & 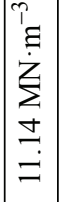 & $\underset{z}{\varangle}$ & 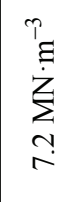 & $\underset{\mathrm{Z}}{\overleftrightarrow{2}}$ & $\overleftrightarrow{\mathrm{Z}}$ \\
\hline $\begin{array}{l}0 \\
0 \\
6 \\
0 \\
0 \\
\underline{x} \\
n \\
n\end{array}$ & $\frac{\varangle}{z}$ & $\begin{array}{l}\vec{j} \\
0 \\
6 \\
0 \\
\underset{n}{\sigma}\end{array}$ & $\underset{z}{\varangle}$ & 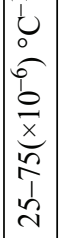 & $\underset{z}{\varangle}$ & 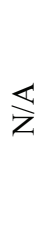 & 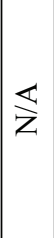 & $\begin{array}{l}0 \\
0 \\
\sigma \\
0 \\
0 \\
x \\
\sigma \\
\sigma\end{array} \mid$ & ¿ & $\underset{z}{\nwarrow}$ & 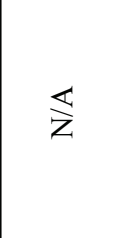 & 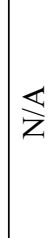 \\
\hline $\begin{array}{l}\text { हี } \\
+\end{array}$ & $\begin{array}{l}\Xi \\
0 \\
\infty \\
0 \\
1 \\
1 \\
\\
\end{array}$ & 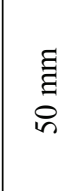 & $\begin{array}{l}\text { E् } \\
\infty \\
\infty \\
\infty\end{array}$ & $\left|\begin{array}{c}\Xi \\
\Xi \\
\infty \\
\text { m }\end{array}\right|$ & $\begin{array}{l}\Xi \\
0 \\
\infty \\
0 \\
i \\
\\
\end{array}$ & $\begin{array}{l}\text { E } \\
\vdots \\
\text { Oల }\end{array}$ & 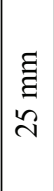 & $\begin{array}{c}\tilde{\Xi} \\
0 \\
+\end{array}$ & $\begin{array}{l}\text { हี } \\
\text { m }\end{array}$ & 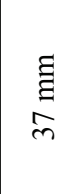 & $\underset{z}{\longleftarrow}$ & $\begin{array}{l}\text { ]ี } \\
\text { m }\end{array}$ \\
\hline 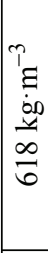 & $\frac{\varangle}{z}$ & 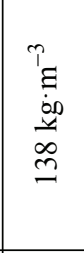 & 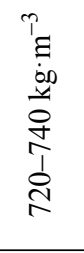 & 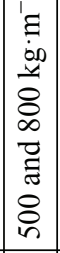 & $\underset{z}{\varangle}$ & 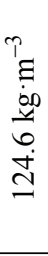 & 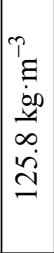 & 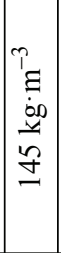 & $\underset{z}{\varangle}$ & 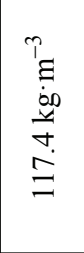 & 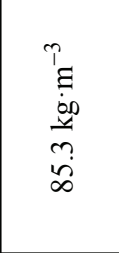 & 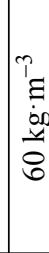 \\
\hline $\begin{array}{l}\overline{0} \\
\overline{0} \\
\overline{0} \\
\overline{0} \\
0 \\
\overline{3}\end{array}$ & 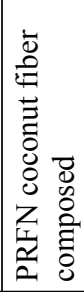 & $\begin{array}{l}\overline{8} \\
8 \\
3 \\
\therefore \\
0 \\
0\end{array}$ & 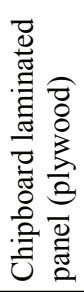 & 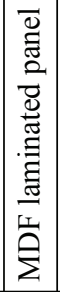 & 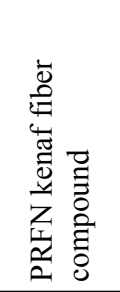 & 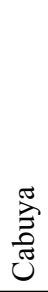 & 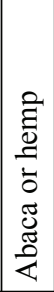 & 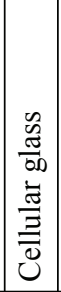 & 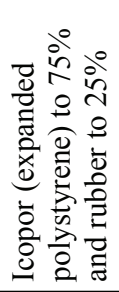 & 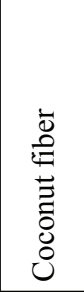 & 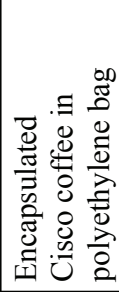 & 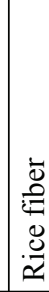 \\
\hline
\end{tabular}


thermal expansion, the less the material will shrink or expand with changes in temperature. A high thermal expansion coefficient affects the dimensional stability of the insulating material (Yuan et al., 2017). In cold conditions, thermal bridges occur as the material shrinks and creates joint cracks or subsidence. This causes a strong impact on the performance of the insulation because it causes premature aging of the same and the need for repair or replacement is essential.

\section{Expert selection}

With the purpose of developing both the Delphi methodology and the AHP methodology, a group of experts was brought together. They established judgments and evaluations of the criteria of applicability and functionality of the insulating materials. In the first assessment, the comparison and weight of the criteria regarding the applicability of the material in prefabricated concrete homes was established, using the Saaty numerical scale. Subsequently, the consistency of said assessments was verified, which provide coherence in the investigation, and consequently, obtained the list of acoustic insulating materials as a result.

\section{Results of the AHP methodology}

The evaluations obtained from the AHP methodology, compares not only the physical-technical properties of the materials, but also criteria regarding the selection of the materials. In other words, the set of evaluations obtained from the AHP methodology helped to generate the list of acoustic materials in descending order with the best options to be applied in manufactured homes. The consistency ratio values with the weights of the criteria and their own vectors once the average of the evaluations and judgments of the experts consulted has been completed. As these values are less than 0.1 , they are useful for the purpose of research.

Table 4 shows the eigenvectors obtained with the evaluations by interviewing the experts, using the Delphi and AHP methodology (eigenvectors of each criteria and the average eigenvector once the necessary iterations have been completed), depending on "availability of the material and price", two criteria with more weight with respect to the applicability of the insulators for the prefabricated homes. This is consistent, because: it is useless for us to have information on a suitable and acoustic insulating material that cannot be found in the market and preferably in the local or national market.

TABLE 4. Eigenvectors of the criteria in AHP methodology

\begin{tabular}{|c|c|c|c|c|}
\hline $\begin{array}{c}\text { Physical-technical } \\
\text { characteristics }\end{array}$ & Availability & Isolation & Installation & $\begin{array}{c}\text { Own vector } \\
\text { (weight of criteria) }\end{array}$ \\
\hline 0.48079291 & 0.080661063 & 0.15691286 & 0.1360679 & 0.213608682 \\
\hline 0.152848742 & 0.199214723 & 0.06714229 & 0.19437598 & 0.153395435 \\
\hline 0.088321967 & 0.457676376 & 0.47875944 & 0.34302058 & 0.341944591 \\
\hline 0.201590302 & 0.158596394 & 0.19099108 & 0.17746785 & 0.182161406 \\
\hline 0.076446079 & 0.103851445 & 0.10619434 & 0.14906768 & 0.108889886 \\
\hline
\end{tabular}


In the same way, the price factor is decisive in the construction environment since more importance is given to lowering prices than to the insulation characteristics. This must change since noise can affect the health conditions of the people on those homes.

The consistency of the responses is less than 0.1 , which translates into coherent answers that provide weight in the investigation to make the list of acoustic insulating materials.

In the evaluations of acoustic materials, several factors were taken into consideration in addition to those mentioned above: the sustainability characteristics of the material, the comparison of existing materials on the market and prototypes proposed in research, since they demonstrate to have an adequate behavior as an insulator / acoustic absorber.

To obtain the result table (Table 5) with the value of the materials: multiply the values of the tables of eigenvectors (functionality of the material) and the weight of the criteria (applicability of the material). Here, the best option is the one with the highest value.

Only materials that are used inside the house are presented in Table 5, since when comparing the values of their characteristics, they differ depending on where the material is applied. For this reason, materials exclusively for internal

TABLE 5. List of sustainable and unsustainable acoustic insulating materials

\begin{tabular}{|l|c|}
\hline $\begin{array}{l}\text { Acoustic insulating / Absorbing } \\
\text { material }\end{array}$ & $\begin{array}{c}\text { AHP } \\
\text { assessment }\end{array}$ \\
\hline Audipet & 0.070696426 \\
\hline Audiseal & 0.050828737 \\
\hline $\begin{array}{l}\text { Sound absorbing plates } \\
\text { (polyurethane foam) }\end{array}$ & 0.049224798 \\
\hline Cork & 0.047869328 \\
\hline Balsa laminated panel & 0.043807815 \\
\hline Curtain & 0.043693331 \\
\hline Audiglue & 0.042399282 \\
\hline Fiberglass & 0.041491311 \\
\hline $\begin{array}{l}\text { PFRV (fiberglass reinforced } \\
\text { plastic) }\end{array}$ & 0.040225609 \\
\hline PRFN composed with jute fiber & 0.034156729 \\
\hline $\begin{array}{l}\text { Veneered chipboard board } \\
\text { - DM board }\end{array}$ & 0.033375732 \\
\hline Polyurethane foam & 0.033366785 \\
\hline Polystyrene foam & 0.033013585 \\
\hline Etherboard or fiber cement & 0.032797451 \\
\hline $\begin{array}{l}\text { Pneumatic acoustic panel } \\
\text { recycled with resin }\end{array}$ & 0.031420276 \\
\hline
\end{tabular}

\begin{tabular}{|l|c|}
\hline $\begin{array}{l}\text { Acoustic insulating / Absorbing } \\
\text { material }\end{array}$ & $\begin{array}{c}\text { AHP } \\
\text { assessment }\end{array}$ \\
\hline Gypsum (plaster) & 0.031133019 \\
\hline Wood fiber & 0.030465064 \\
\hline $\begin{array}{l}\text { PRFN composite with coconut } \\
\text { fiber }\end{array}$ & 0.030142847 \\
\hline Rock wool & 0.029523870 \\
\hline $\begin{array}{l}\text { Chipboard laminated panel } \\
\text { (plywood) }\end{array}$ & 0.028230806 \\
\hline MDF laminated panel & 0.027637440 \\
\hline $\begin{array}{l}\text { PRFN composite with kenaf } \\
\text { fiber }\end{array}$ & 0.027517869 \\
\hline Cabuya & 0.026881398 \\
\hline Abaca or hemp & 0.026365038 \\
\hline Cellular glass & 0.026049431 \\
\hline $\begin{array}{l}\text { Icopor (expanded polystyrene) } \\
\text { at 75\% and rubber at 25\% }\end{array}$ & 0.024062313 \\
\hline Coconut fiber & 0.023963115 \\
\hline $\begin{array}{l}\text { Encapsulated Cisco coffee in } \\
\text { polyethylene bag }\end{array}$ & 0.020250811 \\
\hline Rice fiber & 0.019409784 \\
\hline
\end{tabular}




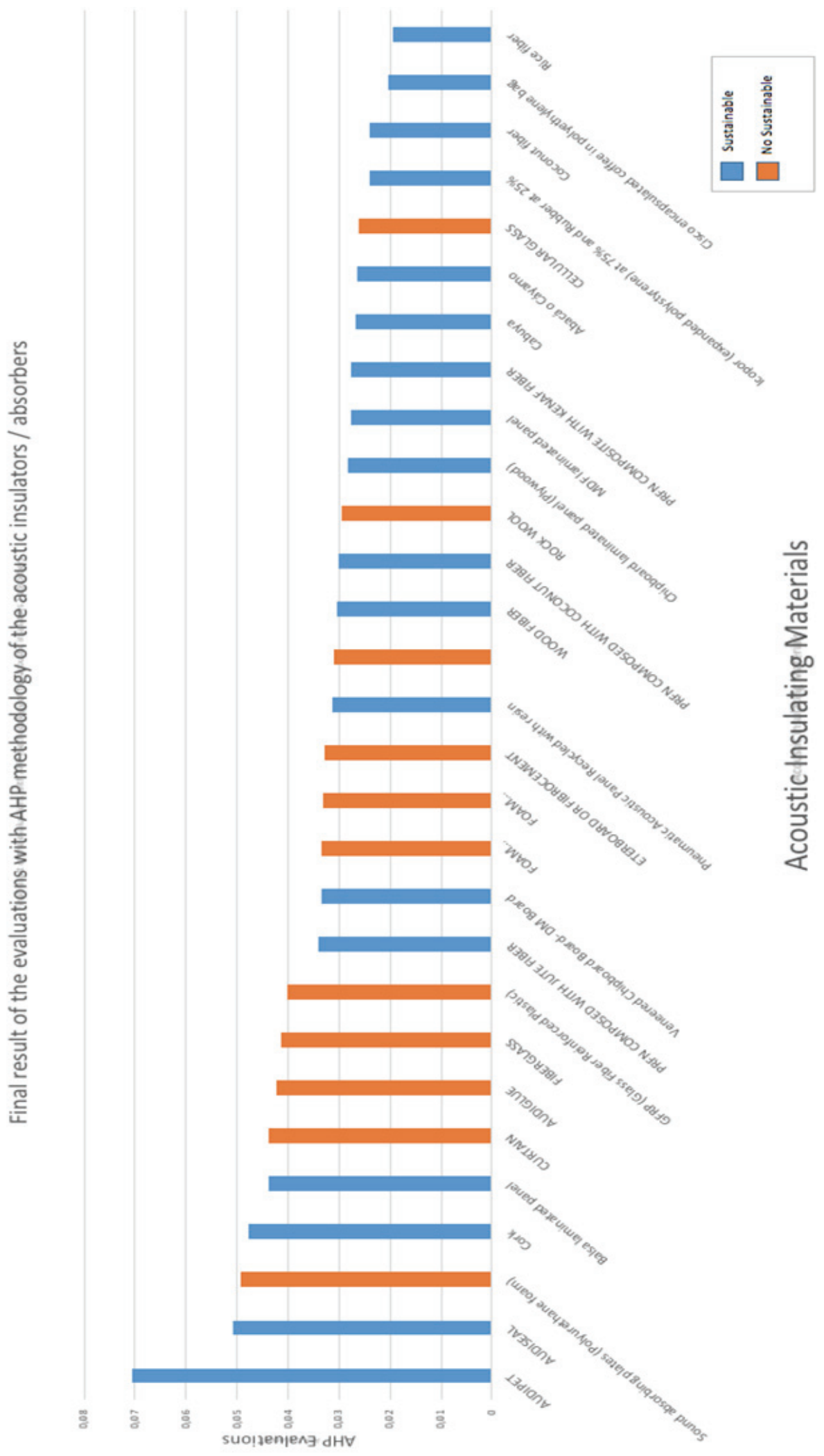

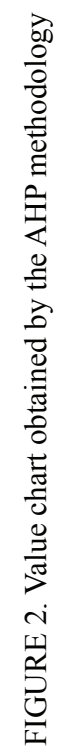


use are compared since the setting and the characteristics of the materials for outdoor use are completely different.

Acoustic insulating materials for ceilings were excluded, although they can be installed inside the building, in precast concrete homes (specifically in modular precast) the mezzanine height standard is $2.40 \mathrm{~m}$, which is not optimal to use it because the living space would be reduced considerably. In Figure 2, the options of the insulators to be used in prefabricated concrete houses are presented in hierarchical order.

In Figure 2, it is evident that the sustainable materials do not present much difference once all the criteria and individual comparisons by criteria have been evaluated. This shows that they are a viable alternative to replace the use of mineral and synthetic materials that are not renewable and that use up the planet's natural resources. The notorious difference in the peaks of the bars is in the criteria of availability, since most sustainable insulators are prototypes. In other words, they are not yet found on an industrial scale, but they prove to be viable and feasible in their realization. In this way, it contributes to an increase in its useful life, to a positive impact on the environment and to the energy efficiency of the home.

\section{Conclusions}

In Ecuador there is no regulation or guide document for the characterization of acoustic materials, since most of them focus on standards for measuring the sound level in indoor and outdoor rooms, in order to later compare the results with the admissible noise levels. Likewise, the sources of information with databases of the materials do not fully specify the physical characteristics of the tested materials (thickness, density, porosity, among others). Consequently, an invitation is made to the acoustic scientific community and interested university groups to organize databases that establish the characteristics of the materials and that serve for a correct comparison of them.

It is worth mentioning that prefabricated materials with additives or resins were not compared to improve acoustic quality in the process plant; because they do not fall into the category of insulating materials to be used once the house is built. However, it is recommended to exclusively develop this type of studies in future research, to improve the quality of the material in terms of insulation and thus, obtaining acoustic comfort without the need to use a material already prepared for this purpose.

To guarantee people's health, acoustic insulation constitutes an important control in the comfort of the home; since in the long run, being subjected to noise pollution translates into health problems. Therefore, it is necessary to implement materials that help mitigate this effect.

In acoustic insulation, plastic reinforced with natural fiber (PRFN) can be classified as alternative materials to plastic reinforced with fiberglass (GRP) because its acoustic absorption coefficient varies between $0.3 \mathrm{NRC}$ for coconut fibers and 0.9 NRC for jute fibers, compared to glass fibers whose absorption was 0.63 NRC. This shows that they have better acoustic absorbent performance. 


\section{References}

Abbass, M. \& Singh, G. (2021). Fatigue analysis of rice husk ash and basalt fibre-based sustainable geopolymer concrete in rigid pavements. Materials Today: Proceedings, 45(6), 5014-5022. https://doi.org/10.1016/ j.matpr.2021.01.450

Banazadeh-Neishabouri, N. \& Shirazi, S.A. (2021). Development of Erosion Equations for Fiberglass Reinforced Plastic (FRP). Wear, 203657. https://doi.org/10.1016/ j.wear.2021.203657

Bao, J., Zou, D., Zhu, S., Ma, Q., Wang, Y. \& Hu, Y. (2021). A medium-temperature, metalbased, microencapsulated phase change material with a void for thermal expansion. Chemical Engineering Journal, 415, 128965. https://doi.org/10.1016/j.cej.2021.128965

Cameselle-Molares, A., Vassilopoulos, A.P. \& Keller, T. (2019). Two-dimensional quasi-static debonding in GFRP/balsa sandwich panels. Composite Structures, 215, 391-401. https:// doi.org/10.1016/j.compstruct.2019.02.077

Caner, H.I. \& Aydin, C.C. (2021). Shipyard site selection by raster calculation method and AHP in GIS environment, İskenderun, Turkey. Marine Policy, 127, 104439. https://doi. org/10.1016/j.marpol.2021.104439

Cevallos, O.A., Jaramillo, D., Ávila, C. \& Aldaz, X. (2017). Production and quality levels of construction materials in Andean regions: A case study of Chimborazo, Ecuador. Journal of Construction in Developing Countries, 22(1), 115-136. https://doi.org/10.21315/ jcdc2017.22.1.7

Chandna, R., Saini, S. \& Kumar, S. (2021). Fuzzy AHP based performance evaluation of massive online courses provider for online learners. Materials Today: Proceedings (in press). https://doi.org/10.1016/j.matpr.2021.02.255

Cheng, A., Lin, W.T. \& Huang, R. (2011). Application of rock wool waste in cement-based composites. Materials and Design, 32(2), 636-642. https://doi.org/10.1016/ j.matdes.2010.08.014

Cherradi, Y., Rosca, I.C., Cerbu, C., Kebir, H., Guendouz, A. \& Benyoucef, M. (2021). Acoustic properties for composite materials based on alfa and wood fibers. Ap- plied Acoustics, 174, 107759. https://doi. org/10.1016/j.apacoust.2020.107759

Dong, H.W., Zhao, S.D., Zhu, R., Wang, Y.S., Cheng, L. \& Zhang, C. (2021). Customizing acoustic dirac cones and topological insulators in square lattices by topology optimization. Journal of Sound and Vibration, 493, 115687. https://doi.org/10.1016/ j.jsv.2020.115687

Escobar, A. (2019). Habitability and design: Radical interdependence and the re-earthing of cities. Geoforum, 101, 132-140. https://doi. org/10.1016/j.geoforum.2019.02.015

García-Ruiz, M.E. \& Lena-Acebo, F.J. (2018). Aplicación del metodo delphi en el diseńo de una investigación cuantitativa sobre el fenómeno FABLAB [Application of the delphi method in the design of a quantitative investigation on the FABLABS]. Empiria. Revista de Metodología de Ciencias Sociales, 40, 129-166. https://doi.org/10.5944/empiria.40.2018.22014

Ghofrani, M., Ashori, A., Rezvani, M.H. \& Arbabi Ghamsari, F. (2016). Acoustical properties of plywood/waste tire rubber composite panels. Measurement: Journal of the International Measurement Confederation, 94, 382-387. https://doi.org/10.1016/j.measurement.2016. 08.020

Guevara Patiño, R. (2016). Estado del arte de lectura del contexto [The state of the art as a research technique: knowledge analysis or quest for new meanings]. Folios, 44(2), 165-179.

He, D., Huang, D. \& Jiang, D. (2021). Modeling and studies of fracture in functionally graded materials under thermal shock loading using peridynamics. Theoretical and Applied Fracture Mechanics, 111, 102852. https://doi. org/10.1016/j.tafmec.2020.102852

José, F., Vidal, L. \& Lluch, A.C. (2019). Diseño y validación mediante Método Delphi de un cuestionario para conocer las características de la actividad física en personas mayores que viven en residencias [Delphy Method validation and design of a questionnaire to assess physical activity]. Retos, 2041(36), 515-520.

Kim, T., Kim, Y. \& Cho, H. (2020). Dynamic production scheduling model under due date uncertainty in precast concrete con- 
struction. Journal of Cleaner Production, 257, 120527. https://doi.org/10.1016/ j.jclepro.2020.120527

Kuranchie, C., Yaya, A. \& Bensah, Y.D. (2021). The effect of natural fibre reinforcement on polyurethane composite foams - a review. Scientific African, 11, e00722. https://doi. org/10.1016/j.sciaf.2021.e00722

Lin, C. \& Kou, G. (2021). A heuristic method to rank the alternatives in the AHP synthesis. Applied Soft Computing, 100, 106916. https://doi.org/10.1016/j.asoc.2020.106916

Liu, Y., Cao, Z., Wang, Y., Wang, D. \& Liu, J. (2021). Experimental study of hygro-thermal characteristics of novel cement-cork mortars. Construction and Building Materials, 271, 121901. https://doi.org/10.1016/j.conbuildm at.2020.121901

Narváez, G. \& León, G. (2001). Caracterización y zonificación climática de la región Andina [Characterization and climatic zoning of the Andean region]. Meteorología Colombiana, 4, 121-126.

Nishimura, T. (2015). Chipboard, oriented strand board (OSB) and structural composite lumber. In Wood Composites (pp. 103-121). Cambridge (UK): Woodhead Publishing. https://doi.org/10.1016/B978-1-78242-4543.00006-8

Obaco, M., Royuela, V. \& Matano, A. (2020). On the link between material deprivation and city size: Ecuador as a case study. Land Use Policy, 104761 (in press). https://doi. org/10.1016/j.landusepol.2020.104761

Opydo, W. (2004). Study of elastic waves of acoustic frequencies generated by surface partial discharges of solid insulators in vacuum. Vacuum, 74(1), 85-92. https://doi. org/10.1016/j.vacuum.2003.12.159

Piuzzi, G.P., Scheuermann Filho, H.C., Villena Del Carpio, J.A. \& Consoli, N.C. (2021). The effects of porosity, asphalt content and fiberglass incorporation on the tensile strength and resilient modulus of asphalt concrete blends. Geotextiles and Geomembranes, 49(3), 864-870. https://doi.org/10.1016/ j.geotexmem.2021.01.002

Plessis, A. du, Babafemi, A.J., Paul, S.C., Panda, B., Tran, J.P. \& Broeckhoven, C. (2021). Biomimicry for 3D concrete printing: a review and perspective. Additive Manufac- turing, 38, 101823. https://doi.org/10.1016/ j.addma.2020.101823

Quiñones-Bolaños, E., Gómez-Oviedo, M., Mouthon-Bello, J., Sierra-Vitola, L., Berardi, U. \& Bustillo-Lecompte, C. (2021). Potential use of coconut fibre modified mortars to enhance thermal comfort in low-income housing. Journal of Environmental Management, 277, 111503. https://doi.org/10.1016/ j.jenvman.2020.111503

Rajeshkumar, V., Anandaraj, S., Kavinkumar, V. \& Elango, K.S. (2020). Analysis of factors influencing formwork material selection in construction buildings. Materials Today: Proceedings, 37(2), 880-885. https://doi. org/10.1016/j.matpr.2020.06.044

Ranaivomanana, H. \& Leklou, N. (2021). Investigation of microstructural and mechanical properties of partially hydrated Asbestos-Free fiber cement waste (AFFC) based concretes: experimental study and predictive modeling. Construction and Building Materials, 277, 121943. https://doi.org/10.1016/j.conbuildm at. 2020.121943

Sato, H., Kurisu, K., Morimoto, M. \& Maeda, M. (2021). Effects of rainfall rate on physical characteristics of outdoor noise from the viewpoint of outdoor acoustic mass notification system. Applied Acoustics, 172, 107616. https://doi.org/10.1016/ j.apacoust.2020.107616

Sivakandhan, C., Murali, G., Tamiloli, N. \& Ravikumar, L. (2020). Studies on mechanical properties of sisal and jute fiber hybrid sandwich composite. Materials Today: Proceedings, 21, 404-407. https://doi.org/10.1016/ j.matpr.2019.06.374

Sreenivasan, S., Sulaiman, S., Ariffin, M.K.A.M., Baharudin, B.T.H.T. \& Abdan, K. (2018). Physical properties of Novel Kenaf Short Fiber Reinforced Bulk Molding Compounds (BMC) for Compression Moulding. In $\mathrm{Ma}$ terials Today: Proceedings, 5, 1226-1232. https://doi.org/10.1016/j.matpr.2017.11.205

Suonox (2019). Audiglue, silence the noise. Monterrey (Mexico): Suonox.

Taban, E., Khavanin, A., Ohadi, A., Putra, A., Jafari, A.J., Faridan, M. \& Soleimanian, A. (2019). Study on the acoustic characteristics of natural date palm fibres: Experimental and theoretical approaches. Building and 
Environment, 161, 106274. https://doi. org/10.1016/j.buildenv.2019.106274

Tang, N., Lei, D., Huang, D. \& Xiao, R. (2019).

Mechanical performance of polystyrene foam (EPS): Experimental and numerical analysis. Polymer Testing, 73, 359-365. https://doi. org/10.1016/j.polymertesting.2018.12.001

Torres, F.G. \& Aragon, C.L. (2006). Final product testing of rotational moulded natural fibre-reinforced polyethylene. Polymer Testing, 25(4), 568-577. https://doi.org/10.1016/ j.polymertesting.2006.03.010

Yuan, J.M., Feng, Y.R., Wu, Z.J., Wang, Y.J., Li, S.Y. \& Sun, P. (2017). A carbon fiber network/polypropylene composite with a low thermal expansion coefficient and high stiffness. Xinxing Tan Cailiao/New Carbon Materials, 32(3), 271-276. https://doi. org/10.1016/j.carbon.2017.06.069

\section{Summary}

Sustainable acoustic insulation for prefabricated concrete homes in Andean zones. The construction sector is undoubtedly one of the main promoters for economic and social development. The modern concept of the prefabrication of elements originated from the industrial revolution to reduce costs and increase production through the mechanization of work, increasing the quality and ease of control in the plant. Prefabricated concrete homes are aimed at the popular sectors, however, when talking about acoustic habitability comfort we refer to constructive solutions used in a building to minimize the transmission of sounds (noise pollution) from outside or from an adjoining enclosure. This is achieved with the use of acoustic insulation materials. In the present research, through a bibliographic review, the updated state-of-the-art search methodology is developed, supported by the Delphi and AHP (hierarchical analytical process) methods and with the opinion of experts to compare the properties of acoustic insulating materials; in order to publicize the best acoustic insulators to be installed inside prefabricated concrete homes. Within the bibliographic review, new technologies with sustainable materials found as feasible prototypes were investigated in Ecuador, where thanks to its diversity of ecosystems and the necessary existing technology, the natural fibers used in the prototypes for insulation/absorption are easily found acoustics in homes.

\section{Authors' address:}

Marcel Paredes - corresponding author

(https://orcid.org/0000-0002-3762-9633)

Universidad Nacional de Chimborazo

Facultad de Ingeniería

Av. Antonio José de Sucre, Riobamba

Ecuador

e-mail: marcelparedes@unach.edu.ec

\section{Alexis Andrade \\ Universidad Nacional de Chimborazo \\ Facultad de Ingeniería \\ Av. Antonio José de Sucre, Riobamba \\ Ecuador}

Universidad Politécnica de Valencia

Camí de Vera, s/n, 46022 Valencia

Espańa
Fausto Lopez
Tito Castillo
Valeria Arroba
Universidad Politécnica de Valencia
Camí de Vera, s/n, 46022 Valencia
Espańa 